\title{
Evaluación de la capacitación de enfermería en dos institutos nacionales de salud.
}

\section{Nursing training assessment in two national institutes of health}

Julio César Cadena Estrada • Sandra Sonalí Olvera Arreola •• María Teresa Pérez López,..•

Carmen L. Balseiro Almario.... Reyna Matus Miranda.....*

\section{Resumen}

Introducción: La capacitación de enfermería es un proceso permanente para adquirir, mantener, renovar, reforzar, actualizar e incrementar los conocimientos, es necesario conocer la opinión del personal respecto a la capacitación impartida por sus instituciones. Objetivo: Evaluar la capacitación del personal de enfermería de dos Institutos Nacionales de Salud. Material y métodos: Estudio observacional comparativo y transversal. La muestra fue aleatoria sistematizada ( $n=415$ enfermeras del área operativa). Se midieron tres dimensiones: planeación, desarrollo y evaluación. Datos recolectados con instrumento ex profeso autoadministrado de 38 ítems sometido a prueba piloto y confiabilidad. Los datos se analizaron con frecuencias, porcentajes, medidas de tendencia central, pruebas T Student, ANOVA de una vía, correlación Pearson y Spearman tomando estadísticamente significativo $p<0.05$. Resultados: La capacitación es evaluada de buena a excelente en las tres categorías laborales y niveles académicos, excepto en las personas que tienen posgrado; el turno que mejor la evalúa es el vespertino (83.34\%); el $80 \%$ del instituto A y $65 \%$ del instituto B evaluaron la capacitación de buena a excelente, lo que se asocia con el nivel académico (rs=0.175, p <0.0001); las especialistas evalúan mejor la planeación que las auxiliares ( $26.0 \pm 7.80, F=4.357, g l=2, p<0.01)$. Discusión: Existe poca evidencia con la cual se puedan comparar los resultados; la mayoría evalúa la capacitación desde la perspectiva de la calidad, aprendizaje o conocimiento. Conclusiones: La evaluación general del proceso de capacitación es considerada por el personal de enfermería como adecuada, lo cual se refleja en la asistencia anual a cursos.

-Licenciado en Enfermería Especialista Cardiovascular. Subjefe de Educación e Investigación en Enfermería. Instituto Nacional de Cardiología., •.Licenciada en Enfermería. Maestranda del Programa de Maestría en Enfermería ENEO UNAM. Jefe del Departamento de Investigación en Enfermería. Instituto Nacional de Cardiología....Licenciada en Enfermería Especialista en Circulación Extracorpórea y Médico Quirúrgica. Maestranda del Programa de Maestría en Enfermería ENEO UNAM. Coordinadora de Investigación en Enfermería. Instituto Nacional de Enfermedades Respiratorias. ...•Doctora en Administración Pública. Profesor de carrera Titular "B" de la ENEO. ......Maestra en Educación. Jefe de la División de Estudios de Posgrado de la ENEO

"Integrantes de la Red de Unidades de Investigación en Enfermería ENEO- Instituciones e Institutos Nacional de Salud. Dirección para correspondencia: Juan Badiano No. 1 Col. Sección XVI, Tlalpan, C.P. 14080, México, D.F.

Correo electrónico: investigacioninc@yahoo.com.mx

FECHA DE RECIBIDO: 16 ENERO 2012 FECHA DE ENVIAdo: 31 MARZO 2012

FECHA DE ACEPTADO: 22 MAYO 2012 


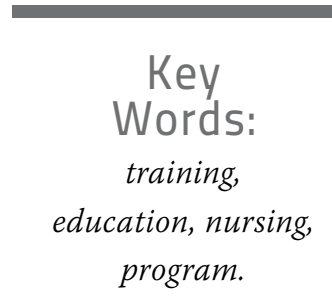

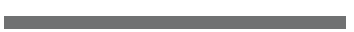

\begin{abstract}
Introduction: Nursing training is a permanent process to acquire, maintain, renew, strengthen, update, and increment knowledge, and because of this, it is necessary to know the staff's opinion about the nursing training offered by their institutions. Objective: To assess the nursing staff training at two National Institutes of Health. Materials and methods: Observational, comparative and basic study. The sample was random and systematized ( $n=415$ nurses from the operative area). Three dimensions were measured: planning, development, and evaluation. Data were collected through a unique self-administered 38 items instrument which was used for a pilot test and further tested for its confidence level. Data were analyzed through frequencies, percentages, central tendency measures, student $T$ tests, one way ANOVA, and Pearson and Spearman correlations, having $p<.05$ as statistically significant. Results: The training is assessed as good-excellent in the three working categories and academic levels, except in the persons who have a post-degree; the shift which better evaluates it is the afternoon one (83.34\%); $80 \%$ from the Institute $A$ and $65 \%$ from the Institute $B$ assessed the training as good-excellent, a finding which is associated with academic level ( $r s=0.175, p<0.0001)$; specialists assess planning better than the auxiliary personnel $(26.0 \pm 7.80, F=4.357, g l=2, p<0.01)$. Discussion: There is little evidence to compare the results; the majority assess the training from the perspective of quality, learning or knowledge. Conclusions: the general assessment of the training process is considered by the nursing staff as adequate, a finding which reflects their assistance to yearly courses.
\end{abstract}

\section{INTRODUCCIÓN}

El proceso educativo acompaña al ser humano desde su origen, por lo tanto es considerado como una acción continua y permanente durante toda la vida, asimismo como parte integral del trabajo. De esta manera, la formación de enfermería constituye la socialización de conocimientos, principios, valores y técnicas en un campo complejo: el cuidado de la salud. Esta formación, en su connotación más amplia es concebida como una posibilidad de autonomía para la creación de proyectos que permitan el desarrollo de su dimensión humana. ${ }^{1}$ Por esta razón la ocupación de estos profesionales y su capacitación son una preocupación universal.

Actualmente, se considera que la educación de enfermería en América Latina ha avanzado en la actualización de sus planes curriculares, los cuales se han desmedicalizado progresivamente en sus programas, en la medida que se ha comprendido el objeto de estudio y la dimensión disciplinar, sin dejar de reconocer que los programas de mayor nivel se encuentran en países y universidades que han desarrollado procesos de autoevaluación y acreditación, así como una cultura de compromiso con la calidad del proceso enseñanzaaprendizaje. ${ }^{2}$ Cabe mencionar que el panorama no es homogéneo, ya que existen programas que se sustentan en el modelo biomédico y en el morbicentrismo, no discuten los avances de la disciplina, ni están a tono con el desarrollo profesional, conservando pedagogías tradicionalistas. ${ }^{3}$ Por otra parte, el panora- ma de la fuerza de trabajo de acuerdo a la Organización Panamericana de la Salud no es nada alentador, ya que sólo el $30 \%$ de enfermeras son profesionales, ${ }^{4}$ lo que de acuerdo con esta organización, tiene un impacto negativo en la calidad y seguridad de la atención.

En las últimas décadas, la globalización y la integración económica de las políticas de capacitación son cada vez más importantes, ${ }^{5}$ así una fuerza laboral bien capacitada es clave para proporcionar a las empresas nacionales, trabajadores con un mayor nivel de calificaciones para adaptarse a los acelerados cambios técnicos del mercado. ${ }^{6}$

En México la construcción del marco regulador de capacitación ha sido progresiva, por ello surge el artículo 123, apartado A, de 
la Constitución Política de los Estados Unidos Mexicanos, ${ }^{7}$ misma que establece que las empresas, cualquiera que sea su actividad, estarán obligadas a proporcionar a sus trabajadores capacitación o adiestramiento para el trabajo; estos preceptos constitucionales son adicionados a la Ley Federal del Trabajo, en su artículo 153 que trata sobre la Capacitación y Adiestramiento de los trabajadores. ${ }^{8}$ Bajo esta perspectiva, el derecho a la capacitación, por un lado, ha originado que las empresas se ocupen del tema; y por otro, que haya instituciones que vigilen la aplicación de lo establecido en esta materia.

La capacitación se define como un proceso activo y permanente que consiste en adquirir, mantener, renovar, reforzar, actualizar e incrementar los conocimientos, las destrezas y las actitudes que le permitan enfrentar y resolver, o en su caso, contribuir a la mejora de los problemas a fin de preservar y mejorar la competencia y actitudes del personal que ha terminado su instrucción formal, ${ }^{9}$ con el objetivo de enfrentar los cambios tecnológicos y de innovación productiva de manera competente y provechosa. De esta manera, cuando se habla de capacitación continua, es preciso entender que el trabajador, cuando asume una actividad profesional, trae un conocimiento previo, resultado de toda su historia de vida y de la preparación profesional formal exigida.

Bajo este contexto, la capacitación representa en sí misma el recurso más importante con el que cuentan las organizaciones, empresas o instituciones, en este caso de salud, para la formación y actualización de sus profesionales. ${ }^{10} \mathrm{Su}$ finalidad es enfrentar la innovación y los cambios tecnológicos de manera competente y provechosa para garantizar el logro de las metas organizacionales de acuerdo con los valores y objetivos de la institución. Para ello, el desarrollo personal de sus integrantes es fundamental, pues se busca mejorar habilidades e incrementar conocimientos en áreas específicas, asimismo, cambiar actitudes con el fin de generar personas proactivas. Por lo tanto, todo personal de salud, en el ejercicio de su profesión, está implícitamente comprometido a la capacitación continua.

Por otro lado, la competitividad de las instituciones se basa cada vez más en la capacidad innovadora, entendida como la potencialidad para efectuar cambios organizacionales, desarrollo de nuevas formas de vinculación con el mercado y aseguramiento de la calidad, todo bajo la premisa de crear ambientes de aprendizaje que propicien confianza, respeto y libertad, y que favorezcan al mismo tiempo mayor involucramiento y compromiso entre los participantes de cada proceso de capacitación, así como el diseño de estrategias de mejora con base en las necesidades específicas de la institución de salud. ${ }^{11}$

Como todo proceso administrativo, la capacitación debe de ser vista como una inversión y un método para lograr el desarrollo de las competencias del personal de enfermería, pues existe una relación directa entre la asistencia a los cursos de capacitación y la mejora de la productividad de enfermería, además de que favorece la disminución de los accidentes o errores de trabajo, se mejora el clima organizacional, la satisfacción en el traba- jo, así como la seguridad y calidad de la atención de los pacientes.

Para que el proceso de capacitación sea ordenado, es necesario seguir varias etapas, a fin de que se estructure la actividad y el aprendizaje. Las etapas que lo conforman son: planeación, desarrollo y evaluación.

Para la planeación es necesario partir de la misión y visión de la organización y tomar en cuenta que los factores relacionados con la participación del personal de enfermería en la capacitación son muy diversos; el grado de participación obedece a razones complejas como las características personales, actitudes, valores, creencias y expectativas. De este modo, los trabajadores asisten a cursos de capacitación como una forma de lograr sus objetivos o metas planteadas de manera personal; por otro lado, hay quienes lo hacen en busca de formar relaciones sociales o mejorar su situación económica; pero muy pocos son quienes se inscriben en busca del conocimiento por su propio valor. ${ }^{12}$

Entre los factores profesionales que más influyen en la capacitación continua se encuentran el turno laboral; a pesar de estar estipulado que la capacitación debe darse dentro de la jornada del trabajador, la realidad es que la mayoría de cursos los proporcionan en el turno matutino. Por lo tanto, existe mayor posibilidad de asistir en los días de descanso o en turno contrario al que se tiene, porque las prestaciones laborales no son las suficientes para el número de días u horas de los cursos de capacitación. ${ }^{9}$

Para el desarrollo de los programas de capacitación las instituciones deben tomar en conside- 
ración varios aspectos: el primero de ellos es la obtención de conocimiento que coadyuve al desarrollo profesional, así como a incrementar las habilidades y destrezas en los procedimientos de una manera mediata, lo que a largo plazo se debe ver reflejado en mejorar la calidad de atención de los usuarios, al haber un cambio de actitudes y aptitudes; el segundo debe estar dirigido a satisfacer las expectativas del personal de enfermería en cuanto a la experiencia, conocimiento y capacidad del profesor para transmitir de una manera clara, explícita y amena el contenido del tema; el siguiente aspecto es la idoneidad de las instalaciones donde se imparte la capacitación, deberán contar con una iluminación adecuada, donde el ruido no sea un motivo de distracción y si es posible que tengan un clima templado, así mismo que el espacio físico esté distribuido de una manera práctica que facilite al asistente desarrollar las actividades académicas adecuadamente; el cuarto aspecto se centra en los recursos didácticos utilizados por el profesor, es bien sabido que por la optimización de tiempo y recursos, las conferencias y similares son la primera opción, sin embargo ofrecen poca retroalimentación y bajos niveles de transferencia y repetición del conocimiento; por último, la duración de la capacitación debe ir acorde con la complejidad y profundidad del tema. ${ }^{13}$

Dentro de la etapa de evaluación están contemplados los beneficios personales que enfermería recibe por capacitarse, está el aprendizaje e interacción con colegas, el intercambio de conocimiento a través de redes sociales, asimismo la posi- bilidad de adquirir una membresía de pertenencia a un grupo donde implícitamente se encuentra la responsabilidad profesional y el desarrollo de la disciplina, claramente relacionado con el conocimiento, las habilidades, la productividad, el mantenimiento de capacidades, la competencia y calidad de la atención. Es indudable que también existirá alguien que asista a cursos para ser acreedor a una compensación económica como estímulo a su capacitación. ${ }^{14}$

La evaluación general del proceso de capacitación debe ser integral, es decir, tomar en cuenta las etapas de planeación, desarrollo y evaluación, para poder tener un juicio de valor que permita identificar el cumplimiento de los objetivos, las áreas susceptibles de mejora, la eficiencia y el desarrollo del potencial del personal de enfermería que ha sido capacitado, un análisis del costo-beneficio, así como los recursos disponibles y su aplicación; el grado de aprendizaje y los resultados en la práctica diaria; aunque también es sustantivo y motivo de este estudio, conocer cómo evalúa el personal de enfermería la capacitación impartida por su institución. ${ }^{14}$

Por otro lado, los Institutos $\mathrm{Na}-$ cionales de Salud, son organismos descentralizados de la Administración Pública Federal, con personalidad jurídica y patrimonio propios que tienen como objeto principal la investigación científica en el campo de la salud, así como la formación y capacitación de recursos humanos de acuerdo a las Condiciones Generales de Trabajo de la Secretaría de Salud, de las cuales se desprende el Reglamento de Capacitación. ${ }^{15}$ Para poder llevar a cabo la misión encomendada y el alcance de los objetivos, debe entre otras cosas, capacitar, adiestrar, así como actualizar periódicamente a los profesionales de enfermería con el propósito de evitar riesgos inherentes a sus actividades; así como favorecer el desarrollo personal para llevar a cabo prácticas de mejoramiento continuo que tiendan al desarrollo de una cultura de calidad y el desarrollo de competencias. La capacitación debe llevarse a cabo con base en un diagnóstico situacional que vincule las necesidades institucionales con las del trabajador, sin embargo, actualmente no todos los programas de capacitación siguen lo anterior, sino que están dirigidos prioritariamente a cubrir las necesidades organizacionales, con frecuencia sin tomar en cuenta, las necesidades del personal de salud.

El personal de enfermería es un elemento clave e indispensable en las instituciones de salud, lo que le obliga a mantenerse actualizado en sus conocimientos para la prestación de sus servicios. Por lo anterior, es preciso que la capacitación se considere el recurso más importante con el cual cuentan las organizaciones de salud para la formación y actualización de sus profesionales. De esta manera, es necesario conocer la opinión que tiene el personal respecto a la capacitación en sus etapas de planeación, desarrollo y evaluación. Con base en lo anterior, se tiene la finalidad de evaluar la capacitación del personal de enfermería de dos Institutos Nacionales de Salud.

\section{Material y métodos}

Se realizó un estudio observacional, comparativo y transversal, con un universo de $\mathrm{N}=1253$ enfermeras 
del área operativa de dos Institutos Nacionales de Salud, de donde se obtuvo una muestra de $n=415$ enfermeras, calculada a través del programa STATs versión 2 para Windows con un intervalo de confianza de $95 \%$, error de 5 y poder de 90\%; la muestra se distribuyó de la siguiente manera: $\mathrm{n}=261$ correspondieron al instituto $\mathrm{A}$ y $\mathrm{n}=154$ al instituto B. La selección de la muestra fue aleatoria sistematizada, para ello, se utilizaron las plantillas de personal de ambas instituciones. Los criterios de inclusión fueron personal de enfermería profesional y no profesional adscrito al área operativa, que se encontraba en el momento de la aplicación de la encuesta, que aceptó participar una vez conocidos los objetivos del estudio y además respondieron la cédula en el $95 \%$ o más.

Para el presente estudio se definió capacitación de enfermería como el proceso activo y permanente que consiste en adquirir, mantener, renovar, reforzar, actualizar e incrementar los conocimientos, las destrezas y las actitudes que le permitan al profesional de enfermería enfrentar y resolver, o en su caso contribuir, a la mejora de los problemas de salud, así como preservar y mejorar la competencia $y$ actitudes del personal de enfermería que ha terminado su instrucción formal.

Dentro del proceso de capacitación de enfermería, se midieron tres variables: planeación, desarrollo y evaluación. Los indicadores estudiados de la primera variable fueron diagnóstico de necesidades, elaboración de programas de capacitación, encuestas sobre capacitación, metas institucionales, congruencia y actualización de los programas; para el desarrollo, se contempló el interés en la capacitación, la obtención de conocimientos teóricos, incremento de habilidades y destrezas, mejoramiento de la calidad, cambio de actitud, desarrollo profesional, competitividad profesional, educación formal, cumplimiento de metas de capacitación, desempeño profesional, continuidad, tiempo y recursos para la capacitación, conocimientos y habilidades de los capacitadores, el ambiente físico y el espacio donde se brinda la capacitación; la evaluación se determinó a través de los beneficios personales, profesionales y económicos del capacitado, la retroalimentación proporcionada, el impacto en la atención del paciente, quejas referentes a la capacitación, la asistencia a cursos de capacitación, evaluación de los resultados, prestaciones institucionales y horarios en que se imparte la capacitación.

Para la recolección de datos se elaboró un instrumento ex profeso autoadministrado con dos apartados: la primera para datos sociodemográficos; y la segunda incluye 38 ítems distribuidos de la siguiente manera: 9 para la dimensión planeación, 17 para desarrollo y 12 para evaluación. Se utilizó una escala de frecuencia, que va desde nunca con valor de 1 hasta siempre con valor de 5 puntos. Los rangos asignados fueron los siguientes: de 1 a 38 puntos, mala capcitación; de 39 a 76, deficiente; de 77 a 114, regular; de 115 a 152, buena; y de 153 a 190 como excelente capacitación. Este instrumento se sometió a prueba piloto y se estimó su confiabilidad a través de Alpha de Cronbach.

Con la información obtenida se estructuró una base de datos en el paquete Statistical Package for the Social Sciences (SPSS) versión 17 para elaborar el análisis a través de estadística descriptiva con frecuencias, porcentajes, media y desviación estándar; y análisis de tipo inferencial con T de Student, correlación de Pearson, Spearman y Anova de una vía, tomando como estadísticamente significativo $\mathrm{p}<0.05$.

$\mathrm{El}$ aspecto ético-legal de la investigación se apoyó en los principios de beneficencia y de respeto a la dignidad humana, el cual incluye el derecho a la autodeterminación y a la información completa.

\section{Resultados}

El instrumento obtuvo un índice de confiabilidad interna a través del Alfa de Cronbach de 0.963. Dentro de los aspectos socio-demográficos predominó el turno matutino con $37 \%$ del personal de enfermería, seguido del vespertino y nocturno con $28 \%$ cada uno; el sexo femenino con un 92\%; la edad osciló entre 31 y 40 años en un $45 \%$; el $88 \%$ eran enfermeras profesionales, de las cuales el 33\% lo ocuparon las licenciadas; prevaleció la categoría de enfermera general con 60\%. El 59\% de los participantes refirieron haber asistido de 1 a 4 cursos de capacitación en el último año.

En cuanto a los participantes que no asistieron a ningún curso en el año, las auxiliares tuvieron el $30 \%$, las generales el 18\% y las especialistas sólo el 14\%; el porcentaje más elevado de enfermeras que acudieron hasta 6 cursos anuales fueron las especialistas con un $83 \%$, seguido de las generales con $75 \%$ y por último las auxiliares con $62 \%$.

La capacitación proporcionada en los dos institutos es evaluada de adecuada a muy adecua- 
da en las tres categorías laborales y niveles académicos, excepto en las personas que tienen posgrado, ya que opinan que la capacitación es de adecuada a regular en un $85.72 \%$ (cuadro 1); y el turno que mejor la evalúa es el vespertino con $83.34 \%$. En la puntuación total, las enfermeras auxiliares evalúan con $93.39 \pm 36.93$; las generales con $95.42 \pm 30.96$; y las especialistas con 106.34 \pm 31.93 .

Con respecto a la evaluación general de la capacitación que se imparten en los dos institutos, el 80.9\% del personal de enfermería del instituto A consideró que la capacitación que recibe es de adecuada a muy adecuada, mientras sólo el $65 \%$ del instituto B opinó lo mismo; en general, el 75\% la consideran de adecuada a muy adecuada (cuadro 2).

El número de cursos a los cuales asistió el personal de enfermería se vio asociado con la planeación $(\mathrm{r}=0.189, \mathrm{p}<0.0001)$, así como con el desarrollo $(\mathrm{r}=0.321, \mathrm{p}<0.0001)$ y la evaluación $(\mathrm{r}=0.408, \mathrm{p}<0.0001)$. Mientras que la evaluación general de la capacitación se asocia con el nivel académico del personal de enfermería en los dos institutos $\left(\mathrm{r}_{\mathrm{s}}=0.175, \mathrm{p}<0.0001\right)$.

El número de cursos a los cuales asiste el personal de enfermería se ve relacionado con la edad $\left(r_{s}=0.129, p=0.009\right)$, no así con la categoría laboral $\left(\mathrm{r}_{\mathrm{s}}=-0.0189\right.$, $\mathrm{p}=0.697)$ y el nivel académico $\left(\mathrm{r}_{\mathrm{s}}=-\right.$ $0.055, \mathrm{p}=0.264$ ).

Al comparar la evaluación general de la capacitación de los dos institutos se encontró que las especialistas evalúan mejor la planeación que las auxiliares; no se observaron diferencias en las otras etapas o categorías laborales (26.0 $\pm 7.80, \mathrm{~F}=4.357, \mathrm{gl}=2, \mathrm{p}<0.01)$. Ocurrió el mismo fenómeno con el nivel académico, donde la mayor puntuación la tuvo la enfermera con posgrado $(X=27.51 \pm 6.85$, $\mathrm{F}=3.09, \mathrm{gl}=4, \mathrm{p}<0.01)$. En relación con el turno vespertino y el especial (cuadro 3) se encontraron diferencias estadísticamente significativas en todas las etapas de la capacitación, donde el turno especial obtuvo las medias más altas (planeación $\mathrm{F}=3.789, \mathrm{gl}=3, \mathrm{p}=<0.01$; desarrollo $\mathrm{F}=5.08, \mathrm{gl}=3, \mathrm{p}=<0.001$; evaluación $\mathrm{F}=4.19, \mathrm{gl}=3, \mathrm{p}<0.05)$.

De igual forma, se encontraron diferencias significativas de cómo evaluó el personal de enfermería la capacitación impartida por sus instituciones en las tres etapas $(\mathrm{t}=-4.94 \mathrm{gl}=413, \mathrm{p}<0.001)$, donde el instituto $\mathrm{B}$ tuvo una media más alta al compararla con el instituto A (106.1 \pm 30.12 vs $90.05 \pm 32.94)$; en el cuadro 4 se pueden observar las medias en cada una de las etapas de la capacitación.

\section{Cuadro 1. Evaluación general de la capacitación de acuerdo a datos laborales del personal de enfermería de dos Institutos Nacionales de Salud.}

\begin{tabular}{|c|c|c|c|c|c|c|c|c|}
\hline \multirow{2}{*}{$\begin{array}{l}\text { Evaluación } \\
\text { de la } \\
\text { capacitación }\end{array}$} & \multicolumn{3}{|c|}{ Categoría laboral } & \multicolumn{5}{|c|}{ Nivel académico } \\
\hline & $\begin{array}{r}\text { Aux. Enf. } \\
\text { Fo (\%) }\end{array}$ & $\begin{array}{r}\text { EG } \\
\text { Fo }(\%)\end{array}$ & $\begin{array}{r}\text { Enf. Esp. } \\
\text { Fo }(\%)\end{array}$ & $\begin{array}{r}\text { Aux. Enf. } \\
\text { Fo }(\%)\end{array}$ & $\begin{array}{r}\text { EG } \\
\text { Fo }(\%)\end{array}$ & $\begin{array}{r}\text { EG Post. } \\
\text { Fo }(\%)\end{array}$ & $\begin{array}{l}\text { Lic. Enf. } \\
\text { Fo (\%) }\end{array}$ & $\begin{array}{l}\text { Posg. } \\
\text { Fo }(\%)\end{array}$ \\
\hline Muy adecuada & $40(37.04)$ & $3(29.44)$ & $10(16.96)$ & $32(35.56)$ & $31(33.70)$ & 18(31.03) & $40(28.57)$ & $2(5.71)$ \\
\hline Adecuada & $40(37.04)$ & $114(45.97)$ & $35(59.32)$ & $35(38.89)$ & $42(45.65)$ & $28(48.28)$ & $62(44.30)$ & $22(62.86)$ \\
\hline Regular & $21(19.44)$ & $47(18.95)$ & $7(11.86)$ & $16(17.78)$ & $15(16.30)$ & $7(12.07)$ & $29(20.71)$ & $8(22.86)$ \\
\hline Poco Adecuada & $5(4.63)$ & $12(4.84)$ & $7(11.86)$ & $5(5.55)$ & $3(3.26)$ & $5(8.62)$ & $8(5.71)$ & $3(8.57)$ \\
\hline Nada adecuada & $2(1.85)$ & $2(0.80)$ & 0 & $2(2.22)$ & 1(1.09) & 0 & $1(0.71)$ & 0 \\
\hline Total & 108 (100) & $248(100)$ & $59(100)$ & $90(100)$ & $92(100)$ & $58(100)$ & 140(100) & $35(100)$ \\
\hline
\end{tabular}

Aux. Enf.= auxiliar de enfermería, EG= enfermera general, Enf. Esp.= enfermera especialista.

EG Post $=$ enfermera general con postécnico, Lic. Enf.= licenciada en enfermería, Posg.= posgrado. 
Cuadro 2. Evaluación de la capacitación de enfermería en dos Institutos Nacionales de Salud.

\begin{tabular}{|c|c|c|c|}
\hline Evaluación de la capacitación & $\begin{array}{r}\text { Instituto A } \\
(\mathrm{n}=261) \\
\text { Fo }(\%)\end{array}$ & $\begin{array}{r}\text { Instituto B } \\
(n=154) \\
\text { Fo (\%) }\end{array}$ & $\begin{array}{r}\text { Total } \\
(\mathrm{N}=415) \\
\text { Fo }(\%)\end{array}$ \\
\hline Muy adecuada & $101(38.7)$ & $22(14.3)$ & $123(29.6)$ \\
\hline Adecuada & $110(42.2)$ & $79(51.3)$ & $189(45.5)$ \\
\hline Regular & $35(13.4)$ & $40(26.0)$ & $75(18.1)$ \\
\hline Poco adecuada & $12(4.6)$ & $12(7.8)$ & $24(5.8)$ \\
\hline Nada adecuada & $3(1.1)$ & $1(0.6)$ & $4(1.0)$ \\
\hline
\end{tabular}

Cuadro 3. Comparación de la media y desviación estándar de la evaluación de las etapas de capacitación por turno.

\begin{tabular}{|c|c|c|c|c|}
\hline \multirow[t]{2}{*}{ Etapas } & \multicolumn{4}{|l|}{$\begin{array}{l}\text { Turno } \\
(\mathrm{N}=415)\end{array}$} \\
\hline & TM & TV & TN & TE \\
\hline Planeación & $23.82 \pm 9.16$ & $21.66 \pm 8.23$ & $24.08 \pm 8.92$ & $27.64 \pm 9.34^{*}$ \\
\hline Desarrollo & $40.07 \pm 5.31$ & $36.23 \pm 3.45$ & $40.90 \pm 4.59$ & $48.12 \pm 20.29$ \\
\hline Evaluación & $33.05 \pm 1.66$ & $30.02 \pm 0.81$ & $34.69 \pm 1.04$ & $36.40 \pm 15.89^{\prime \prime}$ \\
\hline
\end{tabular}

ANOVA de una vía con prueba de Dunnett como Posthoc."p $<0.05$.

$\mathrm{TM}=$ turno matutino, $\mathrm{TV}=$ turno vespertino, $\mathrm{TN}=$ turno nocturno, $\mathrm{TE}=$ turno especial.

Cuadro 4. Comparación de la evaluación por etapas de la capacitación en dos institutos.

\begin{tabular}{llll}
\hline Etapas & $\begin{array}{l}\text { Instituto A } \\
(\mathrm{n}=261)\end{array}$ & $\begin{array}{l}\text { Instituto B } \\
(\mathrm{n}=154)\end{array}$ & $\mathrm{t}$ \\
\hline Planeación & $21.76 \pm 9.07$ & $26.44 \pm 7.88^{*}$ & -5.31 \\
\hline Desarrollo & $37.08 \pm 14.85$ & $44.06 \pm 14.72^{*}$ & -4.64 \\
\hline Evaluación & $31.20 \pm 11.74$ & $35.60 \pm 11.07^{*}$ & -3.76 \\
\hline Evaluación general & $90.05 \pm 32.94$ & $106.10 \pm 30.12^{*}$ & -4.94
\end{tabular}

Prueba T de Student. " $\mathrm{p}<0.05$. 


\section{Discusión}

En el presente estudio se observó que prevalecen en los dos institutos, las enfermeras con título de licenciatura, estos hallazgos concuerdan con lo descrito por Flores Peña et $\mathrm{al}^{9}$, quienes determinaron estadísticamente que el personal de enfermería con estudios de licenciatura le otorga mayor importancia a capacitarse mediante la educación continua. A diferencia de lo anterior, Hernández-Muñoz et al, reportaron que las enfermeras de nivel técnico predominan en la asistencia a cursos de educación continua; también en su estudio observaron que la categoría laboral que predomina en la asistencia a capacitación son las enfermeras generales con un $57 \%,{ }^{16}$ datos semejantes a lo arrojado en esta investigación.

Con respecto a la evaluación de los cursos de capacitación que se imparten en los dos institutos, en general la mayoría del personal de enfermería la considera de buena a excelente, sin embargo el análisis por separado en cada instituto, arroja que en el Instituto A, la mayoría de las enfermeras la evalúa de buena a excelente; mientras que en el Instituto B un gran porcentaje opina que la capacitación es de regular a buena; estos resultados difieren con lo reportado por Valenzuela, donde las ponderaciones obtenidas en su estudio denotan que el personal percibe una capacitación deficiente, ${ }^{17}$ aunque esta percepción, como el mismo autor lo indica, puede estar relacionada a otros factores como tener un segundo trabajo, más de dos hijos y laborar en un turno diferente al matutino.

$\mathrm{Al}$ igual que en esta investigación, Hernández-Muñoz et $\mathrm{al}^{16}$

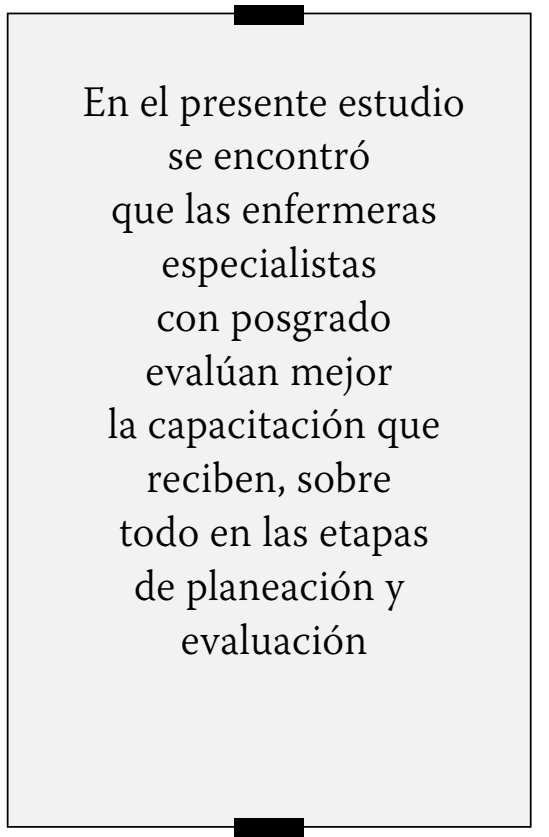

encontraron que el personal de enfermería que más participa en la educación continua es de sexo femenino, cuestión fácil de entender ya que la población de enfermeras a nivel nacional supera el $90 \%$ sobre los hombres; ${ }^{11}$ así mismo el rango de edad que prevaleció fue de 25 a 30 años, datos que difieren con los encontrados en nuestro estudio y con el Instituto Nacional de Estadística, Geografía e Informática que reportó con mayor porcentaje (37.5\%) al rango entre 40 y 49 años. ${ }^{18}$ Cabe destacar que la edad de nuestra población se asoció débilmente con el número de cursos que asisten al año, es decir, mientras tienen más años acuden con mayor frecuencia a cursos. Por otra parte, las enfermeras especialistas con nivel académico de posgrado tuvieron mayor asistencia a cursos.

La mayoría del personal encuestado participó entre 1 a 4 cursos que se imparten en ambos institutos, sin embargo las que menos asisten son las enfermeras auxiliares, seguidas de las generales y en menor porcentaje las especialistas, pero no está asociado con su nivel académico y categoría laboral; estos resultados se asemejan a lo encontrado por Flores Peña et al, ${ }^{9}$ donde los participantes de su estudio asistieron de 2-4 cursos, por lo que se puede suponer que la frecuencia depende de las fechas programadas y de las condiciones del servicio en el cual laboran, tal y como lo reporta Arias-Jiménez M; ${ }^{19}$ en la práctica diaria las enfermeras tienen una baja participación en los programas de capacitación, probablemente debido a que no responden a sus necesidades personales $o$ profesionales.

En el presente estudio se encontró que las enfermeras especialistas con posgrado evalúan mejor la capacitación que reciben, sobre todo en las etapas de planeación y evaluación; dada la suposición de que los programas se realizan con base en un diagnóstico de necesidades del personal, que son en su mayoría enfermeras profesionales, por consecuencia, las auxiliares encuentran temáticas diferentes a sus competencias laborales.

Por otra parte, las enfermeras del turno especial evalúan mejor la etapa de desarrollo, que resultó estadísticamente significativa en comparación con las del turno vespertino, no así con los demás turnos; posiblemente se deba a que las primeras tienen oportunidad de cumplir con el horario completo de los cursos, ya que comúnmente éstos se realizan de lunes a viernes.

Tras la revisión de la literatura, podemos decir que existe poca evidencia con la cual se puedan comparar nuestros resultados, ya que la mayoría de los estudios evalúan la capacitación desde la perspectiva de la calidad del cuidado, del aprendizaje o conocimiento que se 
adquiere tras una intervención de capacitación, o bien desde los factores que favorecen y/o motivan al personal de enfermería para asistir a los cursos; sin embargo se deja a un lado la percepción u opinión que tiene el propio personal sobre las etapas de planeación, desarrollo y evaluación de la capacitación que es impartida en su institución hospitalaria.

\section{Conclusiones}

Se puede concluir que la evaluación general del proceso de capacitación es considerada por el personal de enfermería como adecuada, lo cual se refleja en la asistencia anual a cursos.

La capacitación debe ser vista como una inversión y a sus resultados como una forma de capital, es así que por medio del proceso de capacitación, los profesionales de enfermería y las organizaciones de salud se benefician mutuamente, a partir del resultado de la planeación sistemática de las necesidades de capacitación y el desarrollo del potencial cognoscitivo del personal, así mismo brinda la posibilidad de incrementar y perfeccionar los conocimientos que la organización necesita para su funcionamiento $y$ crea las habilidades y actitudes que se logran por medio del aprendizaje.

Además de conocer las necesidades de capacitación del personal de enfermería y de la propia institución, es necesario desarrollar nuevas estrategias educativas, tales como el aprendizaje basado en problemas, considerando diferentes ritmos de aprendizaje, su experiencia e historia de vida y conocimientos anteriores; así como la planificación del proceso de capacitación a partir de las competencias de en- fermería. Los cursos taller son una buena opción de combinación de la teoría con la práctica con la finalidad de incrementar los conocimientos y la calidad de los procesos de enfermería.

Ante la limitación de evidencias científicas, es necesario profundizar en esta línea de investigación y determinar los principales problemas que visualiza el personal de enfermería en la capacitación e iniciar un cambio en la metodología tradicional.

\section{Agradecimientos}

Agradecemos el apoyo invaluable a la Coordinación de Investigación de la Escuela Nacional de Enfermería de la Universidad Nacional Autónoma de México, para el logro de esta investigación, así como al personal de enfermería que ayudó en la recolección de datos: Alma Elizabeth Alvarez, Francisca Arellano, Marisol Bernal, Martha Castillo, María Mercedes Gallegos, Verónica González, María Guerrero, Gloria Guzmán, Imelda Mendoza, María Isabel Vázquez y Martha Yolanda Vargas.

\section{Referencias Bibliográficas}

1 Chiodelli SN, Lenise PM. Educación continua en el trabajo: Nuevos desafíos para los profesionales de Enfermería. Invest. educ. enferm. 2007;25(1):100-5.

2 Castrillón MC. Pensando en la formación de futuros profesionales de enfermería en América Latina. Inv Educ Enferm. 2008; 26(2):114-21.

3 Rosangela SR, Coelhl S. Educación de enfermería en América Latina: necesidades, tendencias y desafíos. Invest Educ Enferm. 2004;22(2):120-27.

4. Martínez S, Castrillón MC. Over- view of the nursing workforce in Latin America. Washinton: Panamerican Health Organization; 2005 [consultado 18 de Mayo 2010]. Disponible en: http://bit.ly/ ww8CVp

5 Manfredi M. La educación en enfermería: una mirada hacia el próximo milenio. Rev Enferm IMSS. 1999;7(3):151-55.

6 Ballinas-Aguilar A, Gómez-Mejía F. Proyecto-problema: una alternativa de educación continua en la mejora de los servicios de enfermería. Rev Enferm IMSS. 2004; 12(3): 121-2.

7 Carlos R. Notas sobre la Capacitación en México. Rev Latinoamericana de Derecho Social. 2007; 5:165-90.

8 Cámara de diputados del H. Congreso de la Unión [Internet]. México: Ley Federal del Trabajo [actualizado 17 enero 2006; consultado 5 junio 2011]. Disponible en: http:// bit.ly/44AKO

9 Flores-Peña Y, Alonso-Castillo M. Factores que influyen en la participación del personal de enfermería en la educación continua. Rev Latino-am Enfermagem. 2006 [consultado15 junio 2011]; 14(3): [aprox. 8 p.]. Disponible en: http:// bit.ly/UM7lgc

10 Zubizarreta EM. La educación avanzada en enfermería. Realidad y desafíos. Rev Cubana Educ Med Super. 2000;14(3):236-47.

11 Davini MC, Nervi L, Roschke MA. Capacitación del personal de los servicios de salud. Proyectos relacionados con los procesos de reforma sectorial. Quito: Organización Panamericana de la Salud. 2002

12 Werther W, Keith D. Administración de Personal y Recursos Humanos. España: McGraw Hill; 2008. 
13 Oseguera- Torres JP. Actualidad del proceso de capacitación y desarrollo del recurso humano en el Instituto Nacional de Geografía y Estadística (INEGI) [tesis]. Universidad Michoacana de San Nicolás de Hidalgo. Facultad de Contaduría y Ciencias Administrativas; 2010.

14. Siliceo Aguilar A. Capacitación y Desarrollo del personal. 4a ed. México: Limusa; 2004.

15. Condiciones Generales de Trabajo de la Secretaría de Salud. México: Secretaria de Salud. Sindicato Nacional de los Trabajadores al Servicio del Estado; 2012.
16 Hernández-Muñoz A, García-Aguiluz K, Ponce-Gómez G, BernalBecerril ML, Rivas-Espinosa JG. Factores motivacionales en el personal de enfermería que influyen en la asistencia a cursos de educación continua. Rev Enferm IMSS. 2007; 15 (3): 125-128.

17 Valenzuela- Duarte AD, Soto Torres ME, García Pastoressa M, Herrera Pérez LR. Capacitación sobre lactancia materna a personal de enfermería de un hospital de segundo nivel de atención. Revista de Especialidades Médico-Quirúrgicas [Internet]. 2009 [consultado 15 junio 2011];14(2):62-70. Disponible en: http://bit.ly/wkGZei
18 INEGI.Estadísticas a propósito del día de la enfermera. Datos nacionales 2004. [Internet]. México: Instituto Nacional de Estadística y Geografía. 2011. [actualizado 4 enero 2004; consultado 6 enero 2011]. [aprox. 9 pantallas]. Disponible en: http://bit.ly/yPLG6j

19 Arias-Jiménez M. Factores del clima organizacional influyentes en la satisfacción laboral de enfermería, concerniente a los cuidados intensivos neonatales del Hospital Nacional de Niños, 2004. Revista de Enfermería en Costa Rica. Junio 2007; 28(1):12-9. 